\title{
ON A PLANAR EXTERIOR PROBLEM IN LINEAR ELASTICITY
}

BY

H. RAMKISSOON (University of the West Indies )

\begin{abstract}
In this note a representation is obtained for the solution of the planar Dirichlet boundary-value problem of linear elasticity. It consists of a double-layer potential and a linear combination of three basic functions whose form is determined by the shape of the boundary. This representation is similar to that obtained for the analogous problem in hydrodynamics.
\end{abstract}

1. Introduction. In a paper by Korenev [1], a particular representation for the two-dimensional exterior problem of Stokes flow was obtained. The equations of linear elasticity are only slightly more complicated than the governing equations for Stokes flow and in this note it is shown that the treatment of Korenev [1] can be extended to the analogous problem in elasticity.

In the case of plane strain of a homogeneous isotropic elastic body the equilibrium equation is

$$
t_{j i, j}+f_{i}=0
$$

while the constitutive law takes the form

$$
t_{i j}=\lambda u_{\kappa, \kappa} \delta_{i j}+\mu\left(u_{i, j}+u_{j, i}\right) .
$$

Here $t_{i j}$ are components of the stress tensor, $\mathbf{u}$ is the displacement vector, $\mathbf{f}$ is the body force, $(\lambda, \mu)$ are material constants, $\delta_{i j}$ is the Kronecker delta and the indices $(i, j, k)$ take the values 1,2 . Greek indices $(\alpha, \beta)$ will take the values $1,2,3$ and throughout the paper a rectangular co-ordinate system $\left(x_{1}, x_{2}\right)$ will be employed.

The above Eq. (1) and (2) lead to the following basic equation of elasticity:

$$
(\lambda+\mu) u_{\kappa, \kappa i}+\mu u_{i, \kappa \kappa}+f_{i}=0 .
$$

Our objective is to obtain a particular representation for the solution of the homogeneous equation associated with (3) $(f=0)$, in an exterior region $R_{(e)}$ subject to the boundary condition

$$
\lim _{x \in R_{(e)} \rightarrow \xi \in C} \mathbf{u}=\mathbf{a}
$$

\footnotetext{
* Received October 20, 1983.
} 
where $C$ is the boundary. We shall assume that $C$ is a simple closed curve dividing the two-dimensional plane into an interior bounded region $R_{(i)}$ and an exterior unbounded region $R_{(e)}$.

2. Preliminaries. The question of existence and uniqueness of solution of the abovementioned boundary-value problem has been studied extensively, more so by Kupradze [2, 3]. This section is mainly devoted to stating some of the results from these studies that are relevant to our problem.

Consider a concentrated point force applied at the point $y$ in an infinite isotropic elastic plane. This can be represented by

$$
\mathbf{f}=\delta(x-y) \mathbf{e}^{\kappa}
$$

where $\delta(x-y)$ is the Dirac delta function and $\mathrm{e}^{\kappa}$ is the unit vector defined along the $K$ th co-ordinate axis. If we represent the solution of (3) due to this concentrated point force by $\mathbf{u}^{\kappa}$, then the matrix $A(x, y)$ of these so-called fundamental solutions has been shown $[2,4]$ to take the form

$$
A(x, y) \equiv\left[\begin{array}{ll}
u_{1}^{1} & u_{1}^{2} \\
u_{2}^{1} & u_{2}^{2}
\end{array}\right]=\frac{1}{2 \pi}\left[\begin{array}{ll}
\alpha & 0 \\
0 & \alpha
\end{array}\right] \log r+I(x, y)
$$

where $\alpha=(\lambda+3 \mu) / 2 \mu(\lambda+2 \mu), r^{2}=\left(x_{1}-y_{1}\right)^{2}+\left(x_{2}-y_{2}\right)^{2}$ and $I(x, y)$ is a $2 \times 2$ matrix with weak singularities in comparison with the main one. Furthermore, it was shown that

$$
A(x, y)=A^{*}(y, x)
$$

where $A^{*}$ is the transpose of $A$. For convenience, we represent the unknown vector $\mathbf{u}$ in the matrix form

$$
U=\left[\begin{array}{l}
u_{1} \\
u_{2}
\end{array}\right]
$$

and introduce the operators

$$
\begin{aligned}
& T_{1} U=\left\{(\lambda+2 \mu) u_{1,1}+\lambda u_{2,2}\right\} n_{1}+\left\{\mu\left(u_{1,2}+u_{2,1}\right)\right\} n_{2}, \\
& T_{2} U=\left\{\mu\left(u_{1,2}+u_{2,1}\right)\right\} n_{1}+\left\{\lambda u_{1,1}+(\lambda+2 \mu) u_{2,2}\right\} n_{2},
\end{aligned}
$$

where $n$ is the unit outward normal to the curve $C$. If we let

$$
L_{y} A^{*}(x, y)=\left[\begin{array}{ll}
T_{1} A^{* 1} & T_{1} A^{* 2} \\
T_{2} A^{* 1} & T_{2} A^{* 2}
\end{array}\right]
$$

where $A^{* \star}$ are the columns of the matrix $A$, then it can be shown that $[2,4]$

$$
B(x, y)=-\frac{1}{2 \pi}\left[\begin{array}{rr}
\frac{\partial}{\partial n_{y}} & \beta \frac{\partial}{\partial s_{y}} \\
-\beta \frac{\partial}{\partial s_{y}} & \frac{\partial}{\partial n_{y}}
\end{array}\right] \log r+\operatorname{II}(x, y),
$$


where $\operatorname{II}(x, y)$ is a matrix with weaker singularities, $\beta=\mu /(\lambda+2 \mu)$ and $B(x, y)=$ $\left[L_{y} A^{*}(x, y)\right]^{*}$. From these same references $[2,4]$ the following results, stated in Theorems 1-4 can be obtained:

THEOREM 1. The solution of the equation (3) with $\mathbf{f}=0$, has the integral representation

$$
U(x)=\int_{C}\{A(x, y) L(U)-B(x, y) U(y)\} d s_{y},
$$

and the associated Betti formula

$$
\int_{C} U^{*} L(U) d s_{v}=\int_{R_{(i)}} E(U) d R
$$

where

$$
L(U)=\left[\begin{array}{l}
T_{1} U \\
T_{2} U
\end{array}\right]
$$

and $E$ is the internal energy given by $E(U)=(\lambda / 2) e_{i i} e_{j j}+\mu e_{i j} e_{i j}$ with $e_{i j}=\frac{1}{2}\left(\mu_{i, j}+\right.$ $\left.\mu_{j, i}\right)$. The reprersentation (8) suggests the introduction of the single-layer potential

$$
V(x ; \psi)=\int_{C} A(x, y) \psi(y) d s_{y},
$$

and the double-layer potential

$$
W(x ; \phi)=\int_{C} B(x, y) \phi(y) d s_{y},
$$

where the density functions $\psi=\left[\psi_{i}\right]$ and $\phi\left[\phi_{i}\right]$ are column matrices and are assumed to satisfy Hölder's condition.

THEOREM 2. The single-layer potential $V(x ; \psi)$ is continuous throughout the entire plane including $C$.

THEOREM 3. Let $W(\xi ; \phi)_{(i)}$ and $W(\xi ; \phi)_{(e)}$ denote the limiting values of the potential $W(x ; \phi)$ as $x \rightarrow \xi \in C$ from $R_{(i)}$ and $R_{(e)}$ respectively. Then

$$
\begin{gathered}
W(\xi ; \phi)_{(i)}=-\frac{1}{2} \phi(\xi)+\int_{C} B(\xi, \eta) \phi(\eta) d s_{\eta}, \\
W(\xi: \phi)_{(e)}=\frac{1}{2} \phi(\xi)+\int_{C} B(\xi, \eta) \phi(\eta) d s_{\eta} .
\end{gathered}
$$

THEOREM 4. If the operator $L$ is applied to the single-layer potential $V(x ; \psi)$, then

$$
\begin{gathered}
L(V)_{(i)}=\frac{1}{2} \psi(\xi)+\int_{C} L_{\xi} A(\xi, \eta) \psi(\eta) d s_{\eta}, \\
L(V)_{(e)}=-\frac{1}{2} \psi(\xi)+\int_{C} L_{\xi} A(\xi, \eta) \psi(\eta) d s_{\eta} .
\end{gathered}
$$

The systems (10) and (11) are pairwise adjoint and have been used to study the existence and uniqueness of solutions of certain standard boundary-value problems [2]. The use of 
Fredholm alternative for these problems which includes the present problem under investigation, has been justified [2]. We now note the following:

(a) From (9) we have

$$
\int_{C+C_{\tau_{0}}} U^{*} L(U) d s=\int_{R} E(U) d R,
$$

where $C_{\tau_{0}}$ is a circle centered at the orgin (which is taken in $R_{(i)}$ ) and radius $\tau_{0}$ sufficiently large to ensure $C \subset C_{\tau_{0}}$ and $R$ is the bounded region between $C$ and $C_{\tau_{0}}$. It follows by taking the $\lim (12)$ as $\tau_{0} \rightarrow \infty$ that if

$$
\lim _{\tau_{0} \rightarrow \infty} \tau_{0} \int_{0}^{2 \pi} U^{*} L(U) d \theta=0,
$$

then

$$
\int_{C} U^{*} L(U) d s=-\int_{R_{(e)}} E(U) d R .
$$

(b) The equation

$$
\int_{C} U^{*} L(U) d s=0
$$

has three linearly independent solutions $U^{\alpha}$ given by

$$
U^{1}=\left[\begin{array}{l}
1 \\
0
\end{array}\right], \quad U^{2}=\left[\begin{array}{l}
0 \\
1
\end{array}\right], \quad U^{3}=\left[\begin{array}{c}
-X_{2} \\
X_{1}
\end{array}\right] .
$$

(c) From (5) the elements of $A(x, y)$ are of $O(\log r)$. Hence, if the condition $\int_{C} \psi d s=0$ is satisfied for a continuous density function $\Psi$, then the single-layer potential $V(x ; \psi)=O(1 / r)$. In other words,

$$
\lim _{|x| \rightarrow \infty} V(x ; \psi)=0 \quad \text { if } \int_{C} \psi d s=0 .
$$

3. A representation. We shall now generate a representation for the solution of the homogeneous planar exterior problem in elasticity. That is, for the solution of

$$
(\lambda+\mu) u_{k, k i}+\mu u_{i, k k}=0
$$

for $x \in R_{(e)}$, subject to the condition (4) which we shall write as

$$
\lim _{x \in R_{(e)} \rightarrow \xi \in C} U=F(\xi) \equiv\left[\begin{array}{c}
u_{01}(\xi) \\
u_{02}(\xi)
\end{array}\right]
$$

and subject to condition (13) which permits the use of Betti formula in $R_{(e)}$.

LEMma 1. The homogeneous equation

$$
\frac{1}{2} \phi(\xi)+\int_{C} B(\xi, \eta) \phi(\eta) d s_{\eta}=0
$$

has as three linearly independent solutions, the $U^{\alpha}$ 's given by (16). 
Proof. $U^{\alpha}$ satisfies (18) and also $L\left(U^{\alpha}\right)=0$. Substituting into (8), we get

$$
U^{\alpha}(x)=-\int_{C} B(x, \eta) U^{\alpha}(\eta) d s_{\eta}
$$

for $x \in R_{(i)}$. Taking $\lim (21)$ as $x \rightarrow \xi \in C$ and ultilizing (10), produces

$$
U^{\alpha}(\xi)=-W\left(\xi ; U^{\alpha}\right)_{(i)}=\frac{1}{2} U^{\alpha}(\xi)-\int_{C} B(\xi, \eta) U^{\alpha}(\eta) d s_{\eta}
$$

or

$$
\frac{1}{2} U^{\alpha}(\xi)+\int_{C} B(\xi, \eta) U^{\alpha}(\eta) d s_{\eta}=0 .
$$

Hence, $U^{\alpha}(x)$ satisfies (20). Since $U^{1}, U^{2}, U^{3}$ are linearly independent, it means that the $U^{\alpha}$ 's are three linearly independent solutions of (20) and this completes the proof. It follows from Fredholm alternative that the associate equation

$$
\frac{1}{2} \psi(\xi)+\int_{C} L_{\xi} A(\xi, \eta) \psi(\eta) d s_{\eta}=0,
$$

has at least three linearly independent solutions, say $\Psi^{\alpha}$. In fact, we can as in [3], show that they form a complete system of linearly independent set of solutions of (22).

LEMMA 2. The linearly independent set of solutions $\Psi^{\alpha}$ of the homogeneous equation (22) satisfies the condition

$$
\int_{C} \Psi^{\alpha} d s \neq 0
$$

Proof. We form the single-layer potential $V\left(x ; \Psi^{\alpha}\right)$ and observe from (11) that $L\left(V\left(x ; \Psi^{\alpha}\right)\right)_{(i)}=0$. From this result and Betti's formula (9), we see that

$$
\int_{C} V L(V) d s=\int_{R_{(i)}} E(V) d R=0 .
$$

It now follows from (15) and (16) that

$$
V\left(x ; \Psi^{\alpha}\right)=U^{\alpha}, \quad x \in R_{(i)} .
$$

Now assume that $\int_{C} \Psi^{\alpha} d s=0$. From (17), this implies that $\lim _{|x| \rightarrow \infty} V\left(x ; \Psi^{\alpha}\right)=0$. But from (24) and Theorem $1, V\left(x ; \Psi^{1}\right)=U^{1}$ throughout the plane and consequently $\lim _{|x| \rightarrow \infty} V\left(x ; \Psi^{1}\right) \neq 0$. This is a contradiction which means our assumption is false.

LEMmA 3. There exist unique constants $\sigma_{1}, \sigma_{2}$ such that for

$$
\Psi^{4}=\Psi^{3}-\sigma_{1} \Psi^{1}-\sigma_{2} \Psi^{2}
$$

we have

$$
\int_{C} \Psi^{4} d s=0
$$

Proof. We observe that (26) is a system of equations for determining $\sigma_{1}, \sigma_{2}$. To prove that it is uniquely solvable, it suffices to show that the corresponding homogeneous system

$$
\int_{C}\left(\sigma_{1} \Psi^{1}+\sigma_{2} \Psi^{2}\right) d s=0
$$


has only the null solution $\sigma_{1}=\sigma_{2}=0$. If $\sigma_{1} \Psi^{1}+\sigma_{2} \Psi^{2} \neq 0$, then from Lemma 2 ,

$$
\int_{C}\left(\sigma_{1} \Psi^{1}+\sigma_{2} \Psi^{2}\right) d s \neq 0 \text {. }
$$

Therefore, $\int_{C}\left(\sigma_{1} \Psi^{1}+\sigma_{2} \Psi^{2}\right) d s=0$ implies that $\sigma_{1} \Psi^{1}+\sigma_{2} \Psi^{2} \equiv 0$, which in turn implies that $\sigma_{1}=\sigma_{2}=0$. Hence, the proof.

To obtain the required representation, we follow the same procedure as for the Stokes flow in fluid mechanics [1]. We seek a solution of the boundary-value problem (18)-(19) subject to the condition (13), in the form

$$
U(x)=W(x ; \phi)+a^{1} U^{1}+a^{2} U^{2}+a^{3} V\left(x ; \Psi^{4}\right),
$$

where $\Psi^{4}$ is defined in (25). From (10),

$$
F(\xi)-a^{1} U^{1}-a^{2} U^{2}-a^{3} V\left(\xi ; \Psi^{4}\right)=\frac{1}{2} \phi(\xi)+\int_{C} B(\xi, \eta) \phi(\eta) d s_{\eta} .
$$

The constants $a^{\alpha}$ are chosen such that for $\theta=1,2,4$

$$
\int_{C}\left\{F(\xi) a^{1} U^{1}-a^{2} U^{2}-a^{3} V\left(\xi ; \Psi^{4}\right)\right\}^{*} \Psi^{\theta} d s=0 .
$$

To demonstrate the unique solvability of (30) for the $a^{\alpha}$, we consider its homogeneous part

$$
\int_{C}\left\{a^{1} U^{1}+a^{2} U^{2}+a^{3} V\left(\xi ; \Psi^{4}\right)\right\}^{*} \Psi^{\theta} d s=0
$$

This equation (31) is equivalent to the system

$$
\begin{array}{r}
a^{1} \int_{C} \Psi_{1}^{1} d s+a^{2} \int_{C} \Psi_{2}^{1} d s+a^{3} \int_{C} V\left(\xi ; \Psi^{4}\right)^{*} \Psi^{1} d s=0 \\
a^{1} \int_{C} \Psi_{1}^{2} d s+a^{2} \int_{C} \Psi_{2}^{2} d s+a^{3} \int_{C} V\left(\xi ; \Psi^{4}\right)^{*} \Psi^{2} d s=0 \\
a^{3} \int_{C} V\left(\xi ; \Psi^{4}\right)^{*} \Psi^{4} d s=0
\end{array}
$$

From (11) and the already established result $L\left(V\left(x ; \Psi^{\alpha}\right)\right)_{(i)}=0$, it follows that $L\left(V\left(\xi ; \Psi^{4}\right)\right)_{(e)}=-\Psi^{4}$. Using this and (14) in the last equation of (28), we obtain

$$
0=a^{3} \int_{C} V\left(\xi ; \Psi^{4}\right)^{*} \Psi^{4} d s=a^{3} \int_{R_{(e)}} E(V) d R .
$$

Now since $\int_{C} \Psi^{4} d s=0$, we have from (17) $\lim _{|x| \rightarrow \infty} V\left(x ; \Psi^{4}\right)=0$ and so $a^{3} V\left(x ; \Psi^{4}\right)$ vanishes identically in $R_{(e)}$ and on $C$. But since $V\left(x ; \Psi^{\alpha}\right)$ are linearly independent, $V\left(x ; \Psi^{4}\right) \neq 0$. Thus $a^{3}=0$ and (32) simplifies to

$$
\begin{aligned}
& a^{1} \int_{C} \Psi_{1}^{1} d s+a^{2} \int_{C} \Psi_{2}^{1} d s=0 \\
& a^{1} \int_{C} \Psi_{1}^{2} d s+a^{2} \int_{C} \Psi_{2}^{2} d s=0
\end{aligned}
$$


This system (33), is conjugate to that represented by (27) which was in fact shown to possess only the trivial solution. Hence, $a^{1}=a^{2}=0$. We have thus shown that $a^{1}=a^{2}=$ $a^{3}=0$ which proves the unique solvability of (30) for the $a^{\alpha}$ and this in turn guarantees the existence of the solution $\phi$ of (29). These results can be summarized in the following theorem:

THEOREM 5. The solution of the exterior planar boundary-value problem of elasticity characterized by the Eq. (18) and (19) and satisfying the Betti formula (14), is representable in the form

$$
U(x)=W(x ; \phi)+a^{1} U^{1}+a^{2} U^{2}+a^{3} V\left(x ; \Psi^{4}\right),
$$

where $\phi$ is a solution of (29) and the $a^{\alpha}$ are determined from (30).

From this representation, which is similar to that for Stokes flow [1], it is seen that

$$
\mathbf{u}(x)=O(1)
$$

since $W(x ; \phi)=O(1 / r)[3]$.

\section{REFERENCES}

[1] N. K. Korenev, On a representation of the solution of a linearized stationary problem for the Navier-Stokes equations in the case of two space variables, Proc. Steklov Inst. Math. 116, 96 (1971)

[2] V. D. Kupradze, Potential methods in the theory of elasticity, Israel Problem for Scientific Translations, Jerusalem, 1965

[3] V. D. Kupradze, Dynamical problems in elasticity, Progr. in Solid Mech., 3 (1963)

[4] D. Iesan, Existence theorems in the theory of micropolar elasticity, Int. J. Engng. Sci. 8, 777 (1970) 